Supporting Information for:

\title{
Electron and Hole Spin Relaxation in CdSe Colloidal Nanoplatelets
}

Dongmei Xiang, ${ }^{1}$ Yulu Li, ${ }^{1}$ Lifeng Wang, ${ }^{1,2}$ Tao Ding, ${ }^{1}$ Junhui Wang ${ }^{1}$ and Kaifeng $W u^{1 *}$

${ }^{1}$ State Key Laboratory of Molecular Reaction Dynamics and Dynamics Research

Center for Energy and Environmental Materials, Dalian Institute of Chemical

Physics, Chinese Academy of Sciences, Dalian, Liaoning 116023, China

${ }^{2}$ University of the Chinese Academy of Sciences, Beijing 100049, China 


\section{Experimental Methods}

CdSe NPLs were synthesized according literature methods, ${ }^{1,2}$ which are briefly described as follows.

Synthesis of 4 ML CdSe NPLs. In a typical synthesis, $0.18 \mathrm{~g}$ cadmium myristate (Cd(myr)2), $0.012 \mathrm{~g} \mathrm{Se}$, and $15 \mathrm{~mL}$ ODE were degassed under vacuum at $100{ }^{\circ} \mathrm{C}$ for 1 hour. The mixture was then heated to $240{ }^{\circ} \mathrm{C}$ under argon flow. When the temperature reached $200{ }^{\circ} \mathrm{C}, 0.04 \mathrm{~g} \mathrm{Cd}(\mathrm{OAc})_{2} \cdot 2 \mathrm{H}_{2} \mathrm{O}$ were quickly introduced. The reaction continued for $10 \mathrm{~min}$ at $240{ }^{\circ} \mathrm{C}$ before quenched by water bath. When the temperature of the reaction solution was reduced to $160{ }^{\circ} \mathrm{C}, 2 \mathrm{~mL}$ OA was added. After the solution was brought to room temperature, $15 \mathrm{~mL}$ hexane was added. The mixture was then centrifuged at $3000 \mathrm{rpm}$ for $10 \mathrm{~min}$. The precipitate containing a mixture of 3 and 4 ML NPLs was suspended in $30 \mathrm{~mL}$ hexane. The resultant solution was centrifuged at $6000 \mathrm{rpm}$ for $10 \mathrm{~min}$ to remove the 3 ML NPLs as the precipitant.

Synthesis of 5 and 6 ML CdSe NPLs. The two NPLs were both acquired according the following method. In a typical synthesis, $0.17 \mathrm{~g} \mathrm{Cd}(\mathrm{myr})_{2}$ and $14 \mathrm{~mL}$ ODE were degassed under vacuum at $85{ }^{\circ} \mathrm{C}$ for $30 \mathrm{~min}$, and then under argon flow, heated to 250-260 ${ }^{\circ} \mathrm{C}$. $0.012 \mathrm{~g}$ Se well dispersed in $1 \mathrm{~mL}$ ODE with 5-min sonication was quickly injected to the reaction mixture under vigorous stirring. 20-40 s later, $0.06 \mathrm{~g}$ $\mathrm{Cd}(\mathrm{Ac})_{2} \cdot 2 \mathrm{H}_{2} \mathrm{O}$ was added to the flask and the reaction mixture was stirred for another 60 s. $0.15 \mathrm{~mL} 0.5 \mathrm{M}$ cadmium(II) chloride $\left(\mathrm{CdCl}_{2}\right)$ aqueous solution was slowly introduced within a duration of $2 \mathrm{~min}$. After another $3 \mathrm{~min}$, the reaction was quenched by removing the heating mantle. When the temperature was reduced to $160{ }^{\circ} \mathrm{C}$, a 
mixture of $2 \mathrm{~mL} \mathrm{OA}$ and $15 \mathrm{~mL}$ methylcyclohexane (MCH) was added. NPLs were first separated from the reaction batch by centrifugation and were re-dispersed in a smallest amount of $\mathrm{MCH}$. By adding an excessive amount of hexane to $\mathrm{MCH}$, smaller QDs could be separated by centrifugation (NPLs in the precipitants). This step was repeated to increase the purity of NPLs. Remaining precipitants were re-dispersed in $\mathrm{MCH}$ and larger QDs and 5 ML NPLs were removed by size-selective precipitation. This step was also repeated for several times. The final 6 ML NPLs were dispersed in hexane for use. The 5 ML NPLs were also separated from NPLs using size-selective precipitation.

Synthesis of 4 ML CdSe/ZnS NPLs. CdSe/ZnS core/shell NPLs were synthesized following reported procedures with slight modifications. ${ }^{3} 0.2 \mathrm{mmol}$ zinc acetate, $7 \mathrm{~mL}$ 4 ML CdSe NPLs (with optical density of 1.6 at $512 \mathrm{~nm}$ in $1 \mathrm{~mm}$ cuvette), $0.5 \mathrm{~mL} \mathrm{OA}$, and $5 \mathrm{~mL}$ ODE were added to a $50 \mathrm{~mL}$ three-neck flask. The solution was stirred under vacuum at room temperature for one hour to evaporate hexane. The mixture was heated up to $90{ }^{\circ} \mathrm{C}$ and maintained for $30 \mathrm{~min}$ to completely remove water and/or other remaining volatile solvents. After the degassing step, $0.5 \mathrm{~mL}$ oleylamine was added into the solution under nitrogen flow. The reaction was then kept for $10 \mathrm{~min}$ and the temperature was set to $300{ }^{\circ} \mathrm{C}$. Octanethiol-ODE solution $(87.5 \mu \mathrm{L}$ octanethiol in $5 \mathrm{~mL}$ ODE) was prepared in glove box and injected into the reaction at $170{ }^{\circ} \mathrm{C}$ using a syringe pump with a speed of $8.85 \mathrm{~mL} / \mathrm{h}$. When the temperature reached 250 ${ }^{\circ} \mathrm{C}$, the injection speed of octanethiol-ODE solution was changed to $3.54 \mathrm{~mL} / \mathrm{h}$. The reaction was kept at $300{ }^{\circ} \mathrm{C}$ for 60 min and was then quenched by injection of $5 \mathrm{~mL}$ 
ODE and an ice-water bath. The product was washed with $20 \mathrm{~mL}$ ethanol and centrifuged at $5500 \mathrm{rpm}$ for $3 \mathrm{~min}$. The precipitated final product was dispersed in hexane.

Transient absorption. The femtosecond pump-probe TA measurements were performed using a regenerative amplified Ti:sapphire laser system (Coherent; $800 \mathrm{~nm}$, $70 \mathrm{fs}, 6 \mathrm{~mJ}$ per pulse, and $1 \mathrm{kHz}$ repetition rate) as the laser source and a Femto-100 spectrometer (TimeTech LLC) as the spectrometer. The fundamental beam is split into two beams with a beam splitter. The transmitted part was used to pump a TOPAS OPA to generated the pump beam which was chopped at a rate of $500 \mathrm{~Hz}$. And the reflected $800 \mathrm{~nm}$ beam was sent through a delay stage and attenuated with a neutral density filter and focused into a $2 \mathrm{~mm}$ thick sapphire or $\mathrm{CaF}_{2}$ window to generate a white light continuum (WLC) used as the probe beam. The pump and probe beams were focused and overlapped onto the sample. Circularly polarized pump and probe pulses were controlled separately by two sets of broadband polarizing beam splitter cubes (400-700 nm, Thorlabs) and quarter-wave plates $(350-850 \mathrm{~nm}$, Thorlabs).

Time-resolved PL. The femtosecond PL upconversion experiments were performed on a Chimera spectrometer (Light conversion) using a Pharos laser $(1030 \mathrm{~nm}, 100 \mathrm{kHz}$, 230 fs pulse-duration; Light conversion) as the excitation and gate sources. One part of the Pharos output was used to pump an optical parametric amplifier (OPA; TOPAS) to generate the wavelength-tunable excitation pulses, while the other was used as the gate pulse. The light emitted by the sample was collected by lens and focused into a BBO crystal together with the gate pulse to generate the up-converted signal via sum 
frequency generation (SFG). The signal photons were focused onto the entrance slit of a monochromator and detected by the spectrometer. The time-resolved decay curve was obtained by delaying the gate pulse with respect to the pump pulse using a mechanical delay stage. The samples were placed in $1 \mathrm{~mm}$ quartz cuvettes and were vigorously stirred in all the measurements in order to minimize photocharging or damaging of the samples.

\section{Spin relaxation simulation}

As illustrated in Fig. S8a, we consider a spin-polarized state initially prepared by a circularly-polarized photon (denoted as state "A"). For simplicity, the exact quantum numbers are ignored in the figure, and we simply draw the electron and hole in specific “spin-valleys". Hole spin-flip (h-flip, with a rate constant of $k \mathrm{~h}$ ) and electron-hole exchange interaction (EI, with a rate constant of $k_{\mathrm{EI}}$ ) induce inter-conversion between these states. Direct electron-flip via SOC is ignored here because it should be much slower compared to $k_{\mathrm{h}}$ and $k_{\mathrm{EI}}$. The exchange interaction could also be an electron-dangling bond spin mechanism, but it is easy to show the simulated kinetic behavior should be similar to the electron-hole EI.

By using $k_{\mathrm{h}}$ and $k_{\mathrm{EI}}$ as input parameters and solving the coupled kinetic equations, we numerically simulate the time evolutions of the population of $\mathrm{A}, \mathrm{B}, \mathrm{C}$ and $\mathrm{D}$ states. Fig. $\mathrm{S} 8 \mathrm{~b}$ is an example using $k \mathrm{~h}$ of $5 \mathrm{ps}^{-1}$ (i.e., $0.2 \mathrm{ps}$, similar to the measured hole spin-flip time) and $k$ EI of $0.25 \mathrm{ps}^{-1}$ (i.e., $4 \mathrm{ps}$ time constant). A rapidly converts to B 
due to fast hole spin-flip and the two reach a quasi-equilibrium, after which A and B convert to $\mathrm{C}$ and $\mathrm{D}$ via $\mathrm{EI}$.

Experimentally, a co-polarized pump/probe configuration probing at the $\mathrm{HH}$ exciton bleach (XB) measures the sum of the populations of A, B and D, as they all bleach the transition that corresponds to the exciton in A, whereas a counter-polarized pump/probe configuration probing at the $\mathrm{HH} \mathrm{XB}$ measures the sum of the populations of $\mathrm{B}, \mathrm{C}$ and $\mathrm{D}$. The spin relaxation dynamics can then be obtained by performing a subtraction between $[\mathrm{A}+\mathrm{B}+\mathrm{D}]$ and $[\mathrm{B}+\mathrm{C}+\mathrm{D}]$. The simulated results are plotted in Fig. S8c, which indeed reproduces the experimental observation of biphasic decay for spin relaxation probed at the $\mathrm{HH} \mathrm{XB}$, with the fast one caused by hole spin-flip and the slower one caused by EI. Similarly, we can examine the contributions of the A, B, C and $\mathrm{D}$ states to the $\mathrm{LH} \mathrm{XB}$. The $\mathrm{LH} \mathrm{XB}$ only contains contributions from the $\mathrm{CB}$ electron, and hence a co-polarized HH-pump/LH-probe configuration measures the sum of the populations of $\mathrm{C}$ and $\mathrm{D}$ (not $\mathrm{A}$ and $\mathrm{B}$ because of the different selection rules for $\mathrm{LH}$ and $\mathrm{HH}$ bands depicted in Fig. 3a). By performing a subtraction between $[\mathrm{A}+\mathrm{B}]$ and $[\mathrm{C}+\mathrm{D}]$, we obtain the pure electron spin-relaxation dynamics (Fig. S8d) which only contains a slow component. Note that the rate constant of the slow component is actually two times the EI rate constant; this is because there are two channels, with the rate of $k \mathrm{EI}$ for each, leading to the conversion from $\mathrm{A}$ and $\mathrm{B}$ to $\mathrm{C}$ and D (Fig. S8a). 

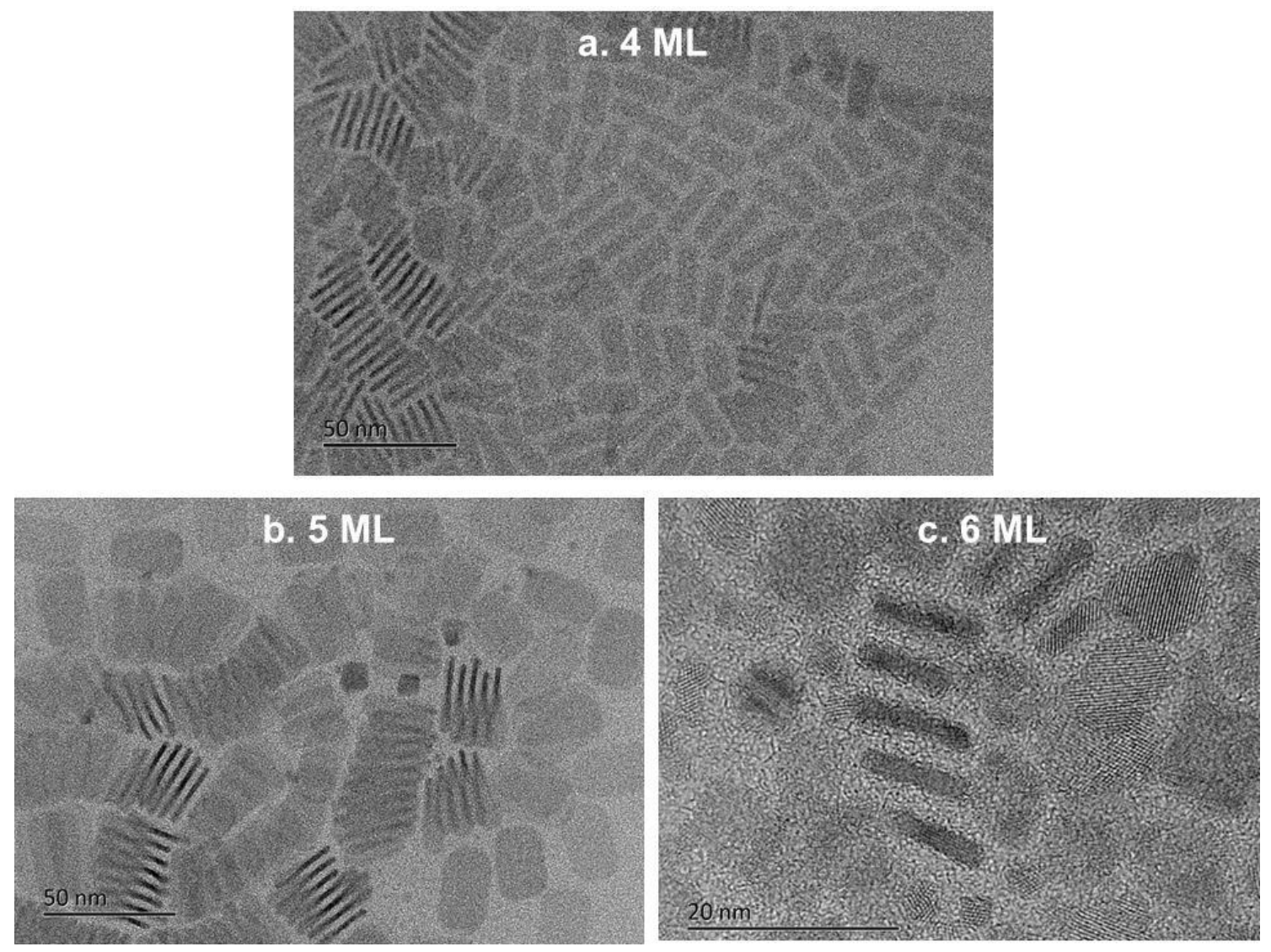

Figure S1. TEM images of (a) $4 \mathrm{ML}$, (b) $5 \mathrm{ML}$ and (c) $6 \mathrm{ML}$ CdSe colloidal NPLs. 

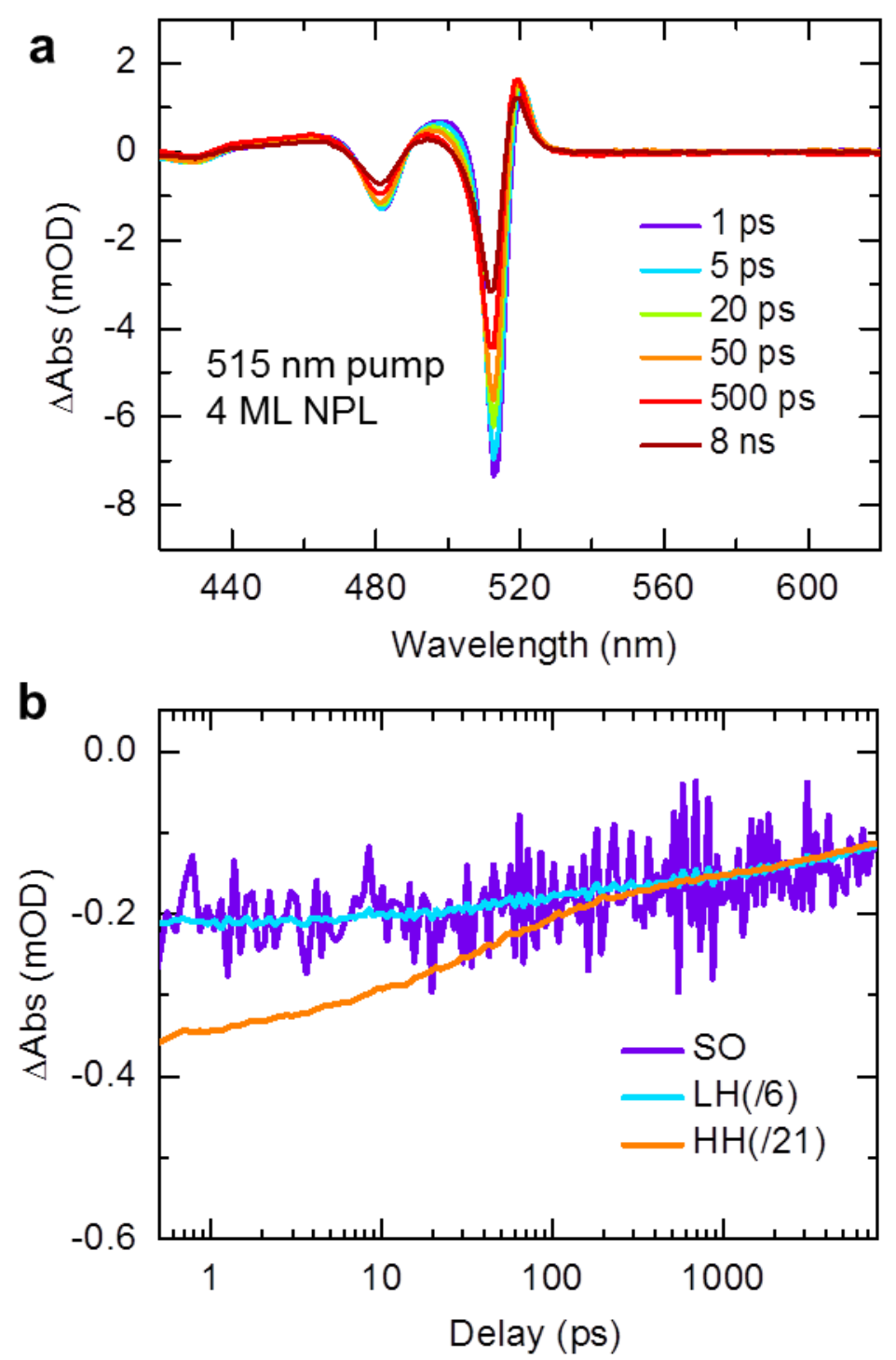

Figure S2. (a) Transient absorption (TA) spectra of 4 ML NPLs at indicated delays following $515 \mathrm{~nm}$ excitation (colored lines). (b) TA kinetics probed at the SO (purple), LH (cyan) and HH (orange) bleach features. The LH and HH traces are divided by indicated factors such as to their long-lived components to that of the SO trace. 

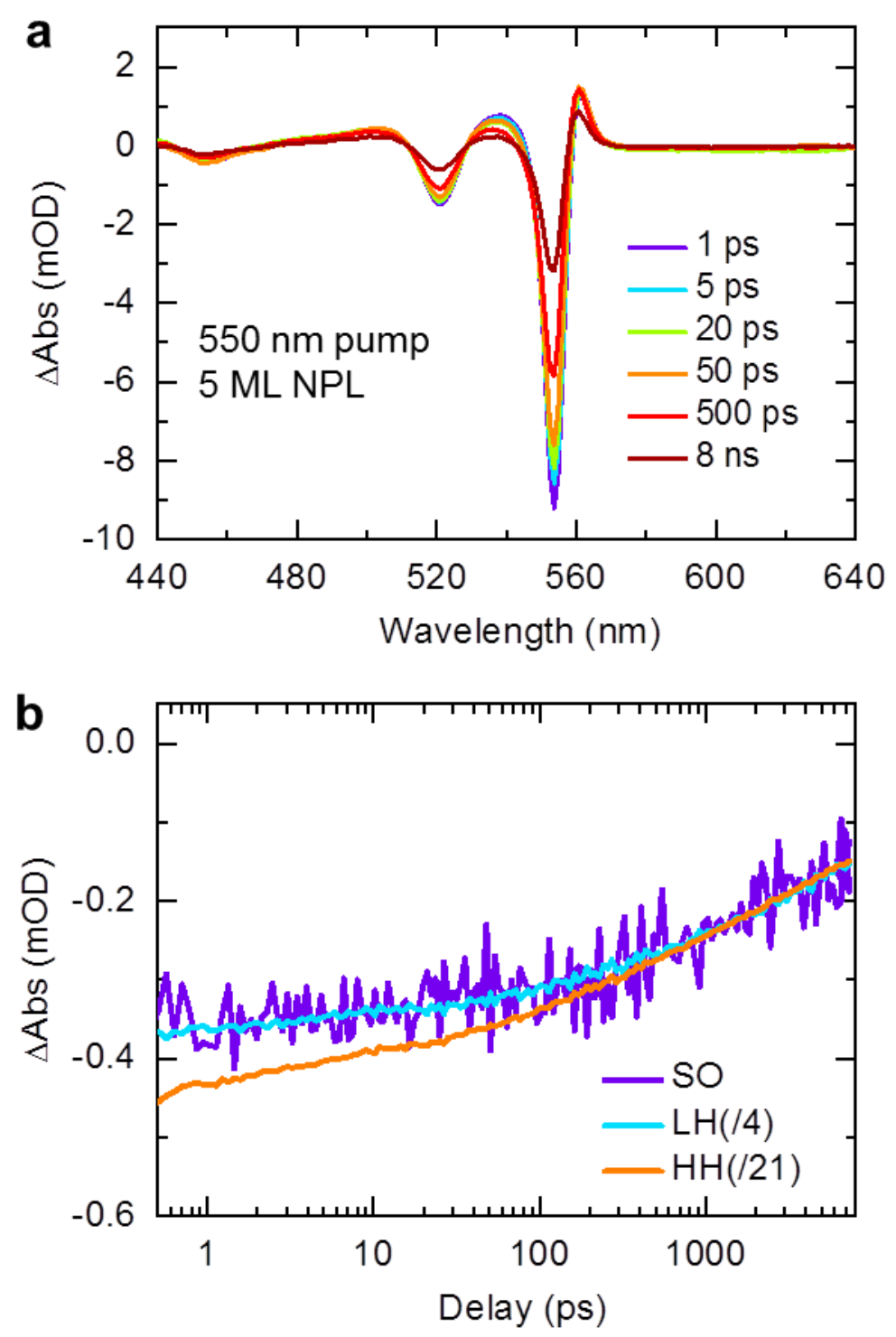

Figure S3. (a) Transient absorption (TA) spectra of 5 ML NPLs at indicated delays following $550 \mathrm{~nm}$ excitation (colored lines). (b) TA kinetics probed at the SO (purple), LH (cyan) and HH (orange) bleach features. The LH and HH traces are divided by indicated factors such as to their long-lived components to that of the SO trace. 
$5 \mathrm{ML} N P L$
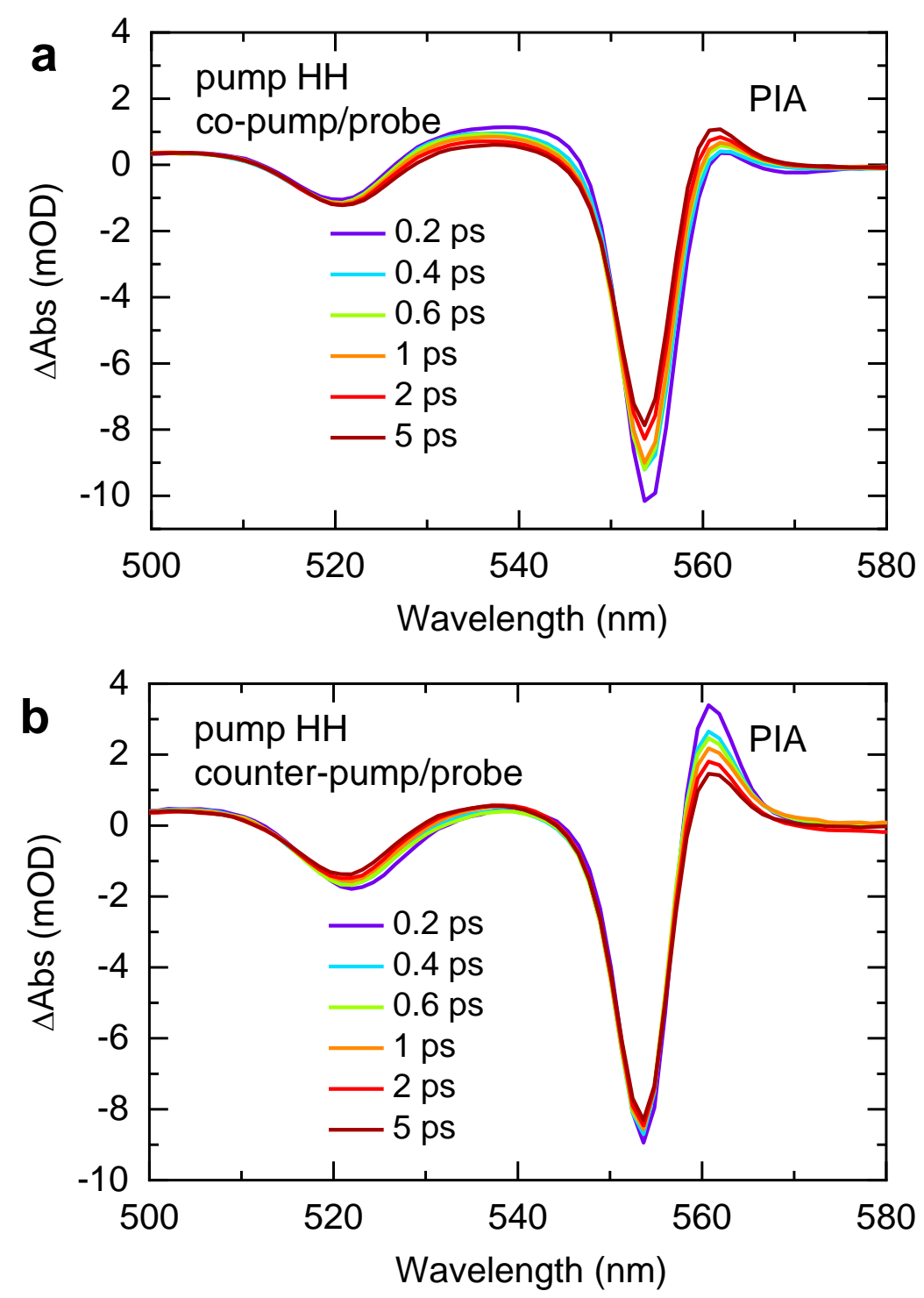

Figure S4. Transient absorption (TA) spectra of 5 ML NPLs at indicated delays following $550 \mathrm{~nm}$ resonant excitation at the $\mathrm{HH}$ exciton, with (a) co- and (b) counter-polarized pump/probe configurations. The photoinduced absorption (PIA) feature on the red side of the HH XB is indicated. 
$5 \mathrm{ML} \mathrm{NPL}$
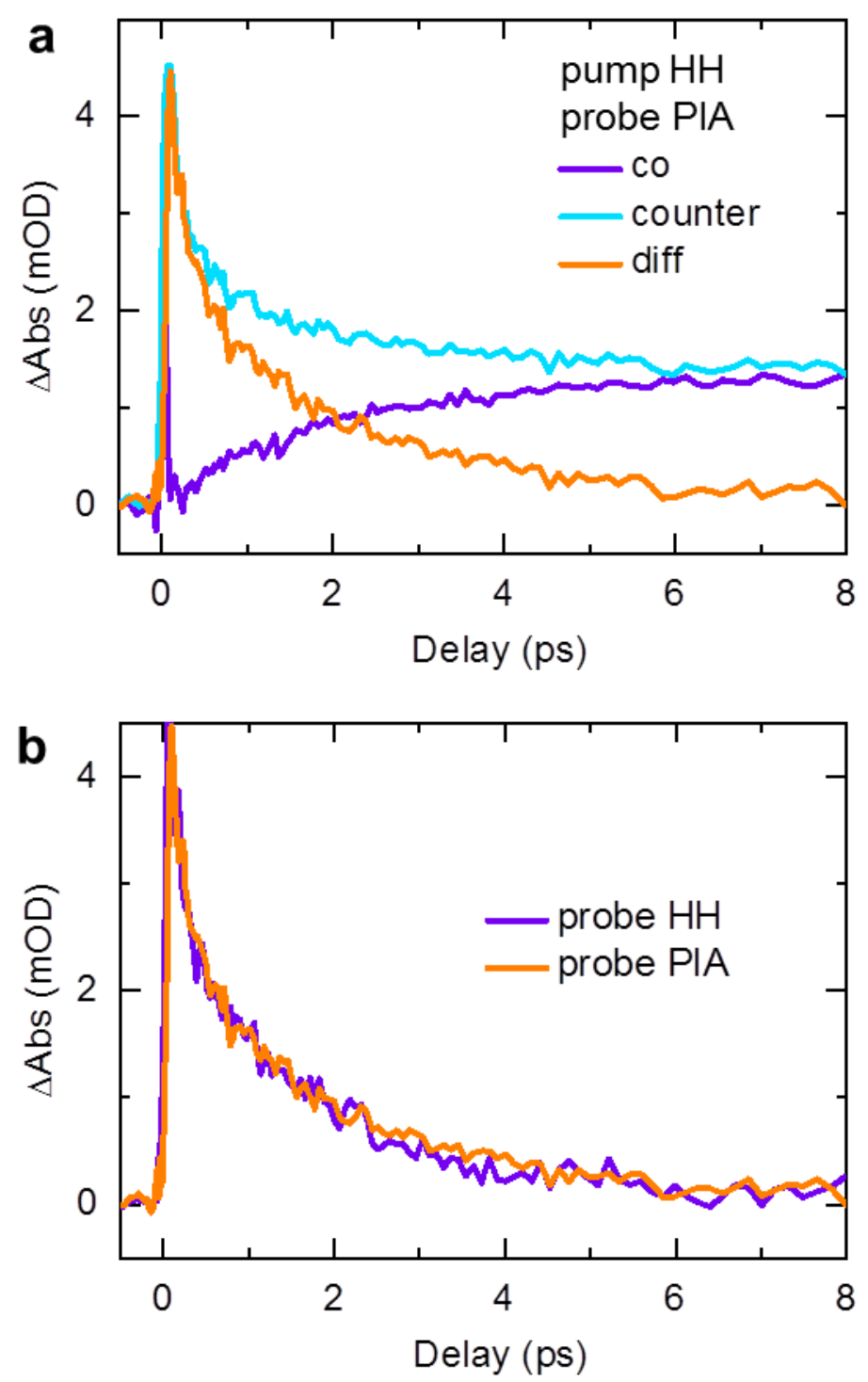

Figure S5. (a) TA kinetics probed at the PIA feature of 5 ML NPLs measured with co(purple) and counter-polarized (cyan) pump/probe configurations and the difference between them (orange) which is a probe of spin relaxation dynamics. (b) Spin relaxation kinetics probed at the $\mathrm{HH}$ (purple) and PIA (orange) features 


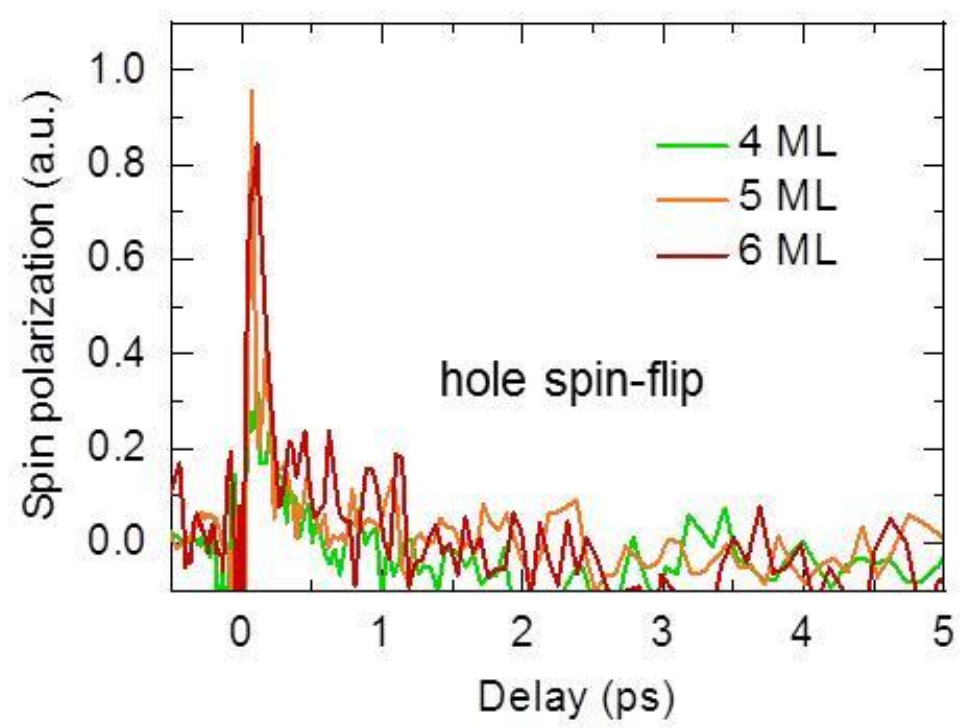

Figure S6. Hole spin-flip kinetics measured for $4 \mathrm{ML}$ (green circles), $5 \mathrm{ML}$ (orange squares) and 6 ML (red triangles) NPLs.

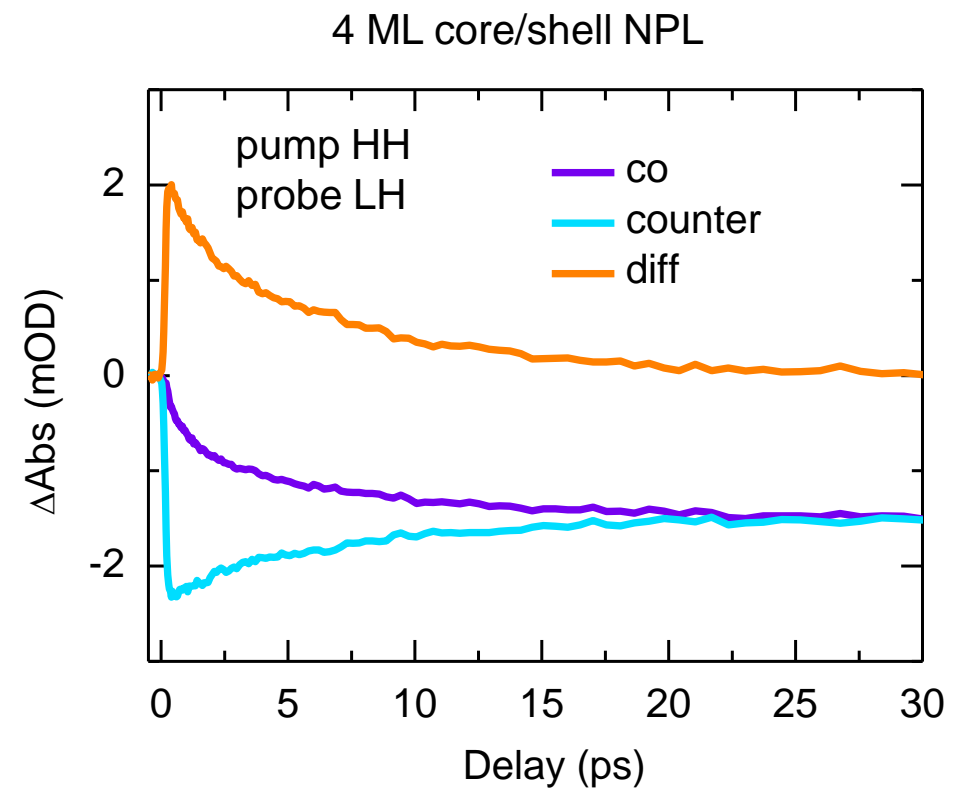

Figure S7. (a) TA kinetics probed at the LH feature of $4 \mathrm{ML} \mathrm{CdSe/ZnS} \mathrm{core/shell}$ NPLs measured with co- (purple) and counter-polarized (cyan) pump/probe 
configurations and the difference between them (orange) which is a probe of electron spin relaxation dynamics.
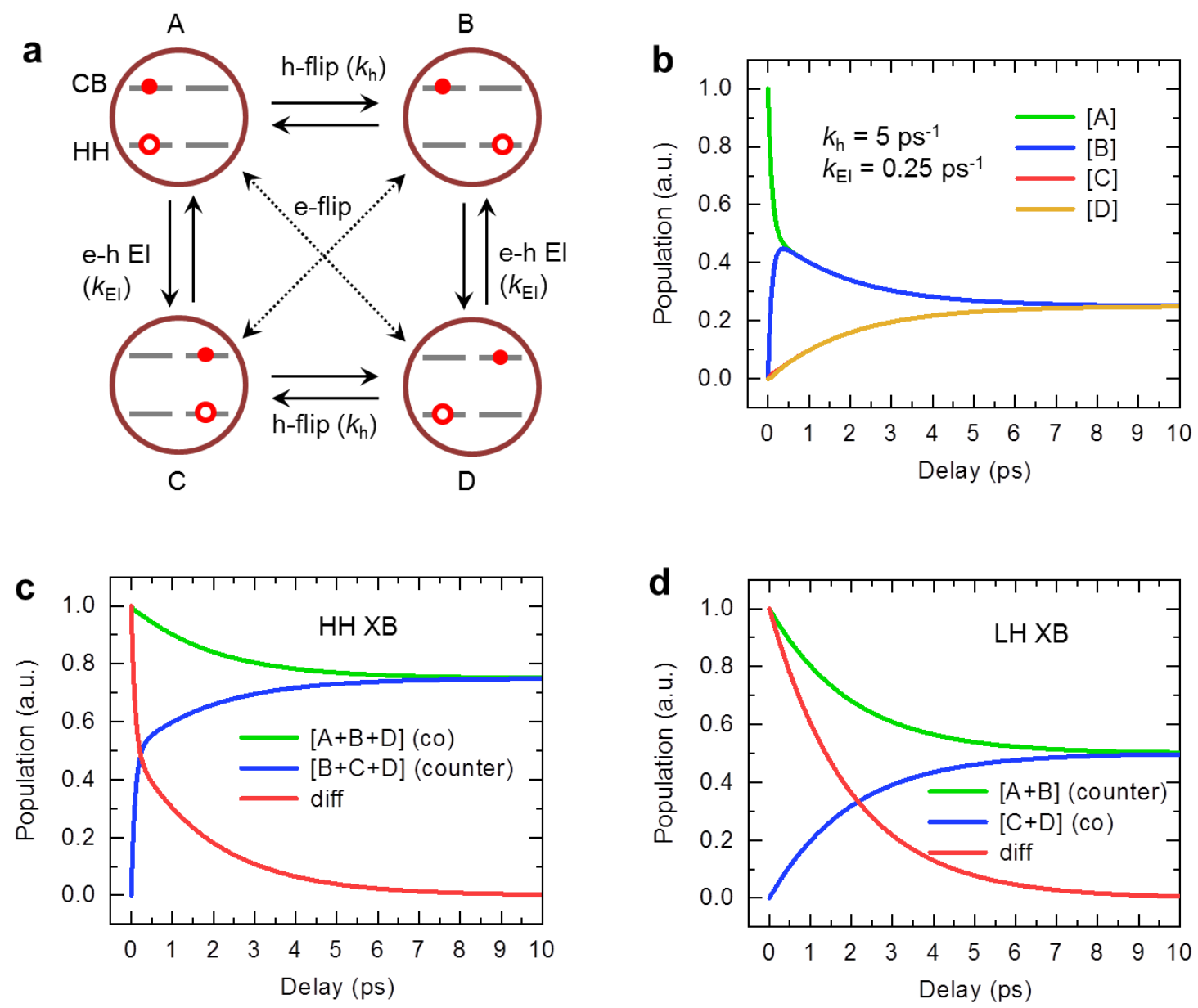

Figure S8. (a) Schematic depiction of inter-conversion between different spin states via hole spin-flip (h-flip, with a rate constant of $k \mathrm{~h}$ ) and electron-hole exchange interaction (EI, with a rate constant of $k_{\mathrm{EI}}$ ) induce inter-conversion between these states. The EI could also be an electron-dangling bond spin mechanism. Direct electron-flip via SOC (e-flip) is ignored here because it should be much slower compared to $k_{\mathrm{h}}$ and $k_{\mathrm{EI}}$. A is initial state prepared by a circularly polarized photon. (b) 
Population evolution for the A (green), B (blue), C (red) and D (orange) states simulated by solving the coupled rate equations using the indicated rate parameters. (c) Simulation of the co- (green) and counter-polarized (blue) HH-pump/HH-probe signals using the concentrations of $\mathrm{A}+\mathrm{B}+\mathrm{D}$ and $\mathrm{B}+\mathrm{C}+\mathrm{D}$, respectively. The difference between them is the spin relaxation dynamics probed at the $\mathrm{HH} \mathrm{XB}$ (red). (d) Simulation of the co- (blue) and counter-polarized (green) HH-pump/LH-probe signals using the concentrations of $\mathrm{C}+\mathrm{D}$ and $\mathrm{A}+\mathrm{B}$, respectively. The difference between them is the spin relaxation dynamics probed at the LH XB (red).

\section{REFERENCES:}

(1) Ithurria, S.; Tessier, M. D.; Mahler, B.; Lobo, R. P. S. M.; Dubertret, B.; Efros, A. L. Colloidal Nanoplatelets with Two-Dimensional Electronic Structure. Nat. Mater. 2011, 10, 936-941.

(2) Cho, W.; Kim, S.; Coropceanu, I.; Srivastava, V.; Diroll, B. T.; Hazarika, A.; Fedin, I.; Galli, G.; Schaller, R. D.; Talapin, D. V. Direct Synthesis of Six-Monolayer (1.9 nm) Thick Zinc-Blende CdSe Nanoplatelets Emitting at 585 nm. Chem. Mat. 2018, 30, 6957-6960.

(3) Altintas, Y.; Quliyeva, U.; Gungor, K.; Erdem, O.; Kelestemur, Y.; Mutlugun, E.; Kovalenko, M. V.; Demir, H. V. Highly Stable, Near-Unity Efficiency Atomically Flat Semiconductor Nanocrystals of CdSe/ZnS Hetero-Nanoplatelets Enabled by ZnS-Shell Hot-Injection Growth. Small 2019, $15,1804854$. 